\title{
Strategies for prevention of ultrasound-guided prostate biopsy infections
}

This article was published in the following Dove Press journal:

Infection and Drug Resistance

8 July 2016

Number of times this article has been viewed

\section{Diane D Lu \\ Jay D Raman}

Division of Urology, Penn State Milton S. Hershey Medical Center, Hershey, PA, USA

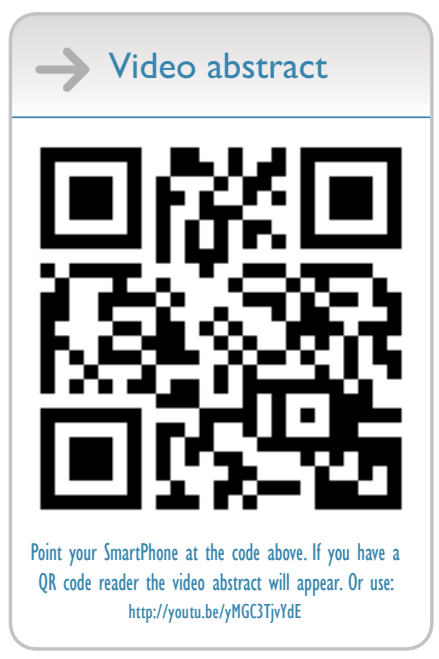

Correspondence: Jay D Raman Division of Urology, Penn State Milton S. Hershey Medical Center, 500 University Drive, Mail Code H055, Hershey, PA 17033-0850, USA

Tel +I 7175316979

Fax + I 7I7 53I 4475

Email jraman@hmc.psu.edu
Abstract: Prostate cancer is the most common cancer in male patients and the second leading cause of cancer-related mortality in males. To confirm the diagnosis of prostate cancer, an ultrasound-guided needle biopsy is necessary to obtain prostate tissue sufficient for histologic analysis by pathologists. Ultrasound-guided prostate needle biopsy can be accomplished via a transperineal or transrectal approach. The latter biopsy technique involves placing an ultrasound probe into the rectum, visualizing the prostate located just anterior to it, and then obtaining 12-14 biopsies. Each biopsy core requires piercing of the rectal mucosa which can inherently contribute to infection. The increasing infectious risk of prostate needle biopsy requires refinement and re-evaluation of the process in which the technique is performed. Such processes include (but are not limited to) prebiopsy risk stratification, antibiotic prophylaxis, use of rectal preparations, and equipment processing. In the subsequent review, we highlight the current available information on different strategies to reduce the risk of infection following prostate needle biopsy.

Keywords: prostate cancer, prostate biopsy, urinary tract infection, sepsis, complications

\section{Introduction}

Prostate cancer is the most common cancer diagnosed among American men; in 2016, $\sim 180,890$ new cases will be diagnosed. The overall trend in mortality is decreasing, however a projected 26,120 men will still die of prostate cancer in 2016. ${ }^{1}$ Prostate needle biopsy (PNB) is the only method of obtaining histologic diagnosis of prostate cancer and for definitively following progression in men on an active surveillance program. While there remains controversy regarding recommendations for screening due to challenges in balancing the perceived treatment-related survival benefit with the likelihood of mortality from all other causes, ${ }^{2} \sim 1.3$ million prostate biopsies are still performed annually. ${ }^{3}$

Infectious complications following prostate biopsy can vary from asymptomatic bacteriuria and bacteremia to frank sepsis, and lead to hospitalization in up to $3.1 \%$ of patients. ${ }^{4-7}$ Average hospitalization time ranges from 5 to 9 days. ${ }^{5,8}$ Recent analysis of the Surveillance, Epidemiology, and End Results (SEER)-Medicare database revealed a 2.65-fold increased risk of overall hospitalization within the first 30 days after prostate biopsy. Furthermore, among those hospitalized for infectious complications, the risk of death within the first month was also significantly higher compared to controls. ${ }^{9}$ Thus, while infectious complications occur in a minority of those undergoing biopsy, the clinical significance can be life-threatening, and judicious selection of patients for biopsy is strongly advised. 
On a global perspective, there has been an increased prevalence of infectious complications and hospitalizations following prostate biopsy in recent years. In the US, the infection rate following PNB increased from $0.4 \%$ to $1.2 \%$ over the period 1991-2007..$^{9}$ In the European Randomized Study of Screening for Prostate Cancer (Rotterdam Section) whereby more than 10,000 prostate biopsies were performed during the period 1993-2011, there was a 10\% increase in hospitalization rate, of which $81 \%$ were due to infections. ${ }^{10}$ Likewise, of more than 75,000 Canadian men undergoing biopsy, there was a $1 \%-4.1 \%$ increase in hospitalization rates over the period 1996-2005 $(P<0.0001)$ and $75 \%$ of admissions were due to infection, though overall mortality rate was unchanged. ${ }^{11}$

In this article, we review the strategies for the prevention of transrectal ultrasound-guided-PNB (TRUS-PNB) infections. We review the technical aspects of biopsy, the periprocedural patient preparation, and prophylactic antimicrobial selection, as well as discuss the current global guidelines in this rising challenge to prevent infectious complications after TRUS-PNB. This is especially relevant in the era of increasing prevalence of fluoroquinolone-resistant Escherichia coli and extended-spectrum $\beta$-lactamase (ESBL)-producing organisms.

\section{Strategies for preventing PNB- related infections \\ Prostate biopsy technique}

Background

TRUS-PNB is performed in the outpatient setting with analgesia via a periprostatic nerve block or under conscious sedation. Some urologists utilize preprocedural enema to help reduce the fecal load for enhanced imaging of the prostate at the time of biopsy, although this is generally considered optional. An 18-gauge needle is inserted through the ultrasound probe which then samples representative sections of the prostate under direct sonographic visualization.

Alternatively, a transperineal approach may be considered. A biopsy grid is utilized to direct the needle into desired regions of the prostate gland using similarly established biopsy templates. One notable difference with the transperineal approach is that the rectal vault is not accessed.

\section{Number of biopsy cores and repeat biopsies}

The 12-core biopsy template is the standard practice advocated by the American Urological Association. ${ }^{12}$ In their extensive review of the literature, they conclude that 12 cores optimally balance the detection rate of clinically significant cancer with acceptable negative predictive values and degree of concordance with the final surgical pathology.

When rising or persistently elevated prostate-specific antigen (PSA) and adverse initial pathology (ie, multifocal highgrade prostatic intraepithelial neoplasia [HGPIN] or atypical small acinar proliferation [ASAP]) are present, repeat biopsy is indicated. In these cases, saturation biopsies (20-24 cores) or an extended template (10-12 cores) plus additional cores from suspicious areas are typically performed. ${ }^{13-15}$

Obtaining a higher number of biopsy cores can theoretically increase the risk of infectious complications, as this would necessitate more passages of the needle through the contaminated rectal mucosa and into the urinary tract. However, studies have been generally inconclusive with regard to effect of this on patient morbidity. No significant increase in febrile complications or hospitalization was found when comparing 6,10 , and 15 cores in a retrospective study of 4,303 men undergoing a total of 5,957 biopsies between 1993 and 2002. ${ }^{16}$ A retrospective analysis of 879 cases of TRUS-PNB in Korean patients, however, found a statistically significant increase in infectious complications in a multiple logistic regression analysis. ${ }^{17}$ However, the range of biopsy cores varied from 4 to 10 , with a mean of 7.8 cores taken and the data may not be generalizable.

In a study of patients undergoing transperineal PNB, hospitalization rate increased from $1 \%$ to $1.6 \%$ as the number of cores expanded from 12 to $>24$. Urinary tract infection (UTI) was the most common indication cited for hospitalization. However, there was a greater proportion of patients undergoing repeat (second or third) biopsy among the subset that underwent extended core biopsies ( 18 or $>24$ cores), which may suggest selection bias and represent an at-risk demographic. ${ }^{18}$ Despite the increased incidence of UTIs, no patients progressed to sepsis in this study. Similarly, in a study of US Medicare patients, repeat biopsy was not associated with increased risk of infectious complications (odds ratio [OR] 0.81, $P=0.39$ ). ${ }^{19}$ Finally, a prospective study has suggested that the number of biopsies does not affect the incidence of bacteriuria or bacteremia. ${ }^{20}$ Collectively, although there is some debate in the literature, the majority of data appears to show no demonstrable increased risk with additional biopsy cores.

\section{Transperineal versus transrectal biopsy}

The transperineal method of obtaining core prostate samples is an attractive approach to reducing postbiopsy infections, as it avoids traversing the rectal mucosa and theoretically reduces the risk of inoculating the urinary tract with gastrointestinal 
flora. This reclassifies the procedure from a contaminated to a clean-contaminated one, whereby the need for broadened prophylaxis is theoretically less of a necessity.

In an Australian series of 245 transperineal biopsies performed on 244 patients, surprisingly, the authors found no readmissions for infectious complications. Only minor complications were observed, including urinary retention (4\%) and clot retention ( $1 \%$ ). However, a weakness of the study was that not all the data were prospectively gathered from individual study centers, thus the true incidence of infectious complications may be underestimated. In addition, biopsy technique was not standardized, and the choice of prophylactic antimicrobial regimen was either heterogeneous or not reported. External validation studies would be necessary to confirm the aforementioned findings. The authors also conducted a systematic review of 16 articles in the published literature between 2003 and 2013 and combining 6,609 pooled patients who underwent transperineal PNB, they found only a $0.076 \%$ readmission rate for sepsis $(n=5) .{ }^{21}$

Prospective randomized studies have shown no difference in complications or cancer detection rate when comparing initial 12- or 14-core prostate biopsy obtained via the transperineal versus transrectal approach. ${ }^{22}$ Patients from both the studies received preprocedural enemas, but they differed in their use of prophylactic antimicrobial regimen, a 3-day course of ciprofloxacin versus single dose of levofloxacin, respectively. ${ }^{21,22}$

While these studies make a convincing argument for the use of the transperineal approach to $\mathrm{PNB},{ }^{21,22}$ certain practical considerations may have hindered its more widespread utilization. This includes the need for general or spinal anesthetic, although some reports of local anesthesia have been reported. Also, the increased technical learning curve and specialized equipment necessary represent additional barriers to utilization. Current guidelines recommend consideration of transperineal approach for obtaining saturation biopsies in men with a previous negative biopsy and in whom there is continued suspicion for cancer, as anterior zone and apical cancers are more easily sampled via this route. ${ }^{23}$

\section{Equipment handling}

Nonpharmacologic modalities aimed to help reduce postPNB infectious complications have been explored to develop an easily reproducible, safe, and cost-effective protocol that avoids the concerns for differing antimicrobial susceptibility and growing resistance patterns. There is concern that the small needle guide used for biopsy may not be amenable to adequate cleaning and therefore serves as a continued nidus for bacterial colonization. These efforts thus focus largely on biopsy probe handling and manufacturing.

Existing research on the utilization of disposable needle guides to decrease infection risk have produced mixed results. The earliest prospective study by Tuncel et al randomized 198 patients to undergo PNB using either a reusable or a disposable needle guide. They showed promisingly that all types of infectious complications (ranging from asymptomatic bacteriuria to febrile UTI) were significantly lower in the latter group $(P<0.001) .{ }^{24}$ However, a smaller prospective randomized study by Gurbuz et al found no difference in infectious outcomes. ${ }^{25}$

Several groups have also evaluated the use of different disinfecting agents to clean the biopsy needle guide. Issa et al studied infection morbidity in 1,642 patients following PNB using probes cleaned with $10 \%$ formalin. Compared to 990 historical controls, the study patients exhibited similar infectious complications, though the results were not statistically significant $(0.8 \%$ versus $0.3 \%$, respectively; $P=0.13) .{ }^{26}$ Similar equivocal outcomes were reported with povidoneiodine in this setting. ${ }^{27}$

Other researchers have proposed coating the biopsy instrument with sustained-release chlorhexidine varnish ${ }^{28}$ to perhaps provide a more durable antibacterial surface. Results from an in vitro model show promising reduction of bacterial growth and infection rates.

\section{Rectal preparation}

Since the bacterial inoculum responsible for PNB-related infections primarily originates in the rectal vault, various rectal disinfecting agents have been evaluated in their ability to reduce bacterial load and subsequent risk of infection.

\section{Enemas}

A survey study in 1998 revealed that $81 \%$ of urologists routinely used prebiopsy enemas, ${ }^{29}$ though the clinical efficacy of reducing infection risk remains debatable. Lindert et al provided one of the first prospective studies where 50 men were randomized to receive either placebo or prebiopsy enema. After obtaining rectal cultures and biopsy specimens, all patients received routine ciprofloxacin and metronidazole (every 12 hours $\times 2$ doses). Their study showed that prebiopsy enema can reduce the incidence of bacteremia from $28 \%$ to $4 \%$, as well as incidence of bacteriuria. ${ }^{20}$

Carey et al performed a retrospective review of 448 TRUS-PNBs, of which 225 patients received sodium bisphosphate enemas prior to biopsy. All patients received 3 days of ciprofloxacin prophylaxis periprocedurally. There was no 
observed difference in clinically significant complications, and routine use of enema before PNB was therefore not recommended. However, two patients in the prebiopsy enema group were hospitalized for complicated UTIs versus none in the control group. ${ }^{30}$ It has been suggested that enemas may cause local irritation to the rectal mucosa and increase risk of bacterial inoculation. ${ }^{31}$

Alternatively, a combined approach of prebiopsy enemas and a 24-hour clear-liquid restricted diet was associated with a reduction in sepsis rate from $2.11 \%$ to $0.46 \%$, though results were not significant $(P=0.89)$. Patients in the control arm were restricted to a clear liquid diet starting the midnight before biopsy. All patients received 7 days ciprofloxacin prophylaxis starting the day prior to the procedure. ${ }^{32}$

\section{Rectal suppository}

A retrospective study by Jeon et al evaluated the efficacy of prebiopsy bisacodyl suppository in 456 patients versus 423 control patients. All patients received either 7 days of cefixime or levofloxacin peribiopsy. Overall infection complication rate was $5.2 \%$, with $3.5 \%$ requiring hospitalization. The authors found that the incidence of infection complication was significantly reduced from $9.5 \%$ to $1.3 \%$ with the use of prebiopsy rectal suppository $(P=0.000) .{ }^{17}$

\section{Rectal cleansing agents}

Povidone-iodine solution, a widely available and cost-effective alternative, has also been studied as a potential rectal cleansing agent. A nonblinded prospective trial by Abughosh et al randomized 865 men to either povidone-iodine prophylactic rectal cleansing or placebo. Both study arms received 3 days of ciprofloxacin prophylaxis starting the day before as well as a Fleets enema 2 hours before PNB. The authors found an overall infectious complication rate of $3.5 \%$. Infection complication was defined as fever $>38^{\circ} \mathrm{C}$, UTI, and/or sepsis. Significant predictors of post-PNB infections were the presence of fluoroquinolone-resistant isolates $(P=0.002)$ and fluoroquinolone exposure in the preceding 3 months $(P=0.009)$. A $42 \%$ risk reduction in infectious complications after povidone-iodine preparation was noted, though it was not statistically significant. ${ }^{33}$

A controlled trial of 100 patients in Thailand randomized to either povidone-iodine rectal cleansing or placebo revealed a reduction in bacteremia from $9 / 50$ to $2 / 50$ study patients $(P=0.025) .{ }^{34}$ Of note, all study patients received prebiopsy enema and ciprofloxacin prophylaxis.

Perhaps, a convincing argument for the use of preprocedural rectal povidone-iodine preparation comes from a retrospective study by Gyorfi et al. ${ }^{35}$ They utilized a slurry consisting of $10 \%$ povidone-iodine solution mixed with lidocaine jelly. All patients received standard prophylaxis, which included either a 3-day regimen of ciprofloxacin or trimethoprim sulfamethoxazole. Researchers observed a reduction in infectious complications from $5.3 \%$ to $0 \%(P=0.03)$ in those receiving standard antibiotic prophylaxis alone versus in combination with the povidone rectal preparation, respectively. Furthermore, $30 \%$ and $18 \%$ of these patients received adjunctive antibiotics at the time of biopsy, respectively. They also noted a significant $97 \%$ reduction in bacterial colony load $(P<0.001)$. This formulation was well tolerated.

\section{Antimicrobial therapy Background}

The most common causative organism in post-PNB infections is E. coli. ${ }^{6,736}$ Additional virulent species include Klebsiella pneumoniae, Pseudomonas aeruginosa, Enterococcus species, Enterobacter cloacae, and Staphylococcus epidermidis..$^{7,37-39}$ The beneficial role for antibiotic prophylaxis is reflected in one of the earliest studies which showed that the routine administration of periprocedural antibiotic reduced the rate of bacteriuria from $20 \%-36 \%$ to $8.6 \%{ }^{40}$ Reported bacteremia was as high as $55 \%$ among those who did not receive routine prophylaxis. ${ }^{41}$

Fluoroquinolones are the most commonly prescribed prophylactic antimicrobial regimen utilized in those undergoing prostate biopsy as it provides broad coverage against these common causative species. In addition, fluoroquinolones have been shown to provide excellent penetration of prostatic tissue in quantities that parallel or exceed concentrations achievable in the serum. ${ }^{42}$

When guideline recommendations on antimicrobial prophylaxis for prostate biopsy were still in infancy, a multicenter study by Kapoor et al provided level I evidence to show the efficacy of single-dose ciprofloxacin in reducing bacteriuria rate from $8 \%$ to $3 \%(P=0.009)$. In this study, $2 \%$ of untreated patients required hospitalization for febrile UTI compared to none of those who received prophylaxis. ${ }^{39}$

Further support for prophylaxis came from trials comparing infectious complications following administration of placebo $(n=75)$ versus single dose $(n=79)$ or 3 -day series $(n=77)$ of combination ciprofloxacin and tinidazole. Researchers found that infectious complications were significantly higher in the placebo group compared to those receiving combination prophylaxis $(P=0.003) .{ }^{38}$

\section{Summary of current standard practice guidelines}

The American Urological Association Best Practice Policy Statement recommends antimicrobial prophylaxis for all 
patients undergoing prostate biopsy, assigning a Ib level of evidence grading. Empiric agents of choice include either a fluoroquinolone or a first-/second-/third-generation cephalosporin. Alternatively, trimethoprim-sulfamethoxazole or an aminoglycoside (or aztreonam if renal insufficiency exists) may also be considered. Duration of therapy is $\leq 24$ hours. If prebiopsy rectal swab cultures are obtained, then prescription of culture-directed antibiotic is advised. ${ }^{43}$

The Canadian Urological Association, however, offers less specific guidelines. Citing findings of the 2011 Cochrane Review, ${ }^{44}$ a grade Ia level of evidence was given for the use of antibiotic prophylaxis in transrectal prostate biopsy. Urologists are advised to consider local antibiograms in selecting appropriate antibiotic regimen, with consideration of obtaining rectal swab cultures preprocedurally for patients at high risk of colonization with multidrug-resistant flora. While the Canadian Urological Association also recognized the equivalent effectiveness of single dose versus short-/longcourse antibiotics, a specific recommendation on treatment duration was not given. ${ }^{45}$

The European Association of Urology recommends quinolones as the prophylactic drug of choice for PNB. The 2015 European Association of Urology guidelines acknowledge the concern for rising fluoroquinolone resistance, though no specific recommendation is made in prevention approach. ${ }^{46}$ The updated 2016 guidelines focus on nonantibiotic prevention strategies and specifically give a grade $\mathrm{B}$ recommendation for the use of povidone-iodine rectal cleansing in addition to standard antimicrobial prophylaxis if a patient is at high risk of infectious complications. Role for preoperative urine culture is uncertain. The European Association of Urology meta-analysis revealed no associations between number of biopsy cores or use of local anesthetic and infectious complications. No recommendations were made regarding the PNB route or utilization/handling of prostate biopsy needles. ${ }^{47}$

\section{Short-course versus extended-course antibiotics}

Multiple trials have shown equivalent outcomes in patients receiving single-dose or extended-course antimicrobial prophylaxis for PNB. A prospective randomized trial showed that 1- versus 3-day regimen of levofloxacin can be administered safely with equivalent efficacy, ${ }^{48}$ and similar results were reported elsewhere. ${ }^{49}$

\section{Targeted antibiotic therapy based on prebiopsy rectal swab cultures}

Tailoring antimicrobial prophylaxis to the patients' microbiome has the potential to reduce the use of broad-spectrum antibiotics and reduce infection rates. Several studies have implemented a pre-PNB rectal swab culture to help guide selection of appropriate antimicrobial prophylaxis. Duplessis et al found no evidence of post-PNB infection complications among 235 men who received targeted prophylaxis when compared to 3/103 historical controls. Prevalence of fluoroquinolone resistance was $14 \%$. The only significant predictor of antibiotic resistance was age, whereas previous antibiotic use within the last year or previous biopsies were not. Targeted prophylaxis was also cost-effective. ${ }^{50}$ The cohort in a study by Taylor et al also did not develop any infectious complications after the institution of targeted prophylaxis, and they found that 38 males required treatment to prevent one infection. This equated to a cost savings of nearly US $\$ 4,500$ per infection prevented. ${ }^{51}$ However, the decision to utilize rectal swab culture was left to the discretion of the urologist, thus there is concern for selection bias.

Some more recent studies have shown less convincing support for the utilization of targeted prophylaxis. Dai et al performed a retrospective nonrandomized study of 317 patients receiving targeted prophylaxis and 173 patients receiving 3 days of ciprofloxacin. While there was a decreased OR (0.7) of post-PNB infection in the targeted prophylaxis group, the results were not significant. There was also no difference observed in the incidence of infectious complications between the two groups. ${ }^{52}$

A prospective study performed at a Veterans Affairs medical center in Utah showed that patients with fluoroquinolone-resistant organisms were significantly more likely to have a previous history of PNB or positive urine culture within the last year ( $P=0.032$ and 0.018 , respectively). While the overall infection rate decreased from $2.8 \%$ to $0.6 \%$ with targeted prophylaxis, the result was not significant. A limitation of this study was that empiric prophylaxis differed among providers and historical controls were utilized. ${ }^{53}$

A retrospective multicenter study by Liss et al on 5,355 patients undergoing PNB showed no difference in the incidence of sepsis following the start of targeted prophylaxis. ${ }^{54}$ However, $25 \%$ of urologists prescribed augmented prophylaxis in the empiric group, which may have potentially lowered the inherent sepsis rate in the control group. In addition, $88 \%$ of men who received appropriate antimicrobial therapy based on preprocedural rectal swab still progressed toward sepsis. Thus, other virulent factors must play a role. Nonetheless, while this study showed that the infection complication rate may not be improved upon with targeted prophylaxis, it does promote judicious antibiotic use safely without over treating patients undergoing PNB. 
Larger randomized prospective studies will provide data to draw more valid conclusions in the role for targeted antibiotic prophylaxis in PNB based upon prebiopsy rectal swab cultures. From the infection cost standpoint, this practice is justified in the literature. ${ }^{55,56}$ Urologists may consider targeted prophylaxis in those with risk factors for colonization with bacterial strains resistant to standard antibiotics.

\section{Special considerations Recent antibiotic use}

Recent antibiotic use prior to biopsy has been shown in multiple studies to increase infectious complication rate, mainly due to drug-resistant bacteria. Akduman et al retrospectively compared sepsis rates among males who received long-term fluoroquinolones versus placebo after prostate biopsy. ${ }^{57} \mathrm{~A}$ total of 558 men were referred for biopsy based on an abnormal digital rectal exam or PSA. The first group consisted of 205 males who received levofloxacin $500 \mathrm{mg}$ daily for 3 weeks, and the second group of 353 males did not receive any extended antibiotic therapy prior to biopsy. All patients received perioperative levofloxacin $500 \mathrm{mg}$ once daily starting a day prior to biopsy for a total of 6 days. Approximately 5.4\% and $1.7 \%$ of patients in groups 1 and 2 , respectively, required hospitalization for postbiopsy sepsis, and this was statistically significant $(P=0.0297)$. Overall, ten of the 17 septic patients had positive blood and/or urine cultures, with E. coli being the most common agent identified (nine patients). One patient had concomitant E. coli and methicillin-resistant Staphylococcus aureus bacteremia. Resistance patterns among E. coli isolates were as follows: $100 \%$ to fluoroquinolones, $66.7 \%$ to trimethoprim-sulfamethoxazole, and $66.7 \%$ to gentamicin. Approximately $55.6 \%$ of isolates were ESBL positive.

A prospective study evaluating septic complications showed similar findings. ${ }^{58}$ All 913 patients in this study received standard empiric prophylaxis consisting of ciprofloxacin $500 \mathrm{mg}$ twice a day $\times 4$ days starting the day prior to biopsy. Overall sepsis rate was $2.8 \%$ (26 patients), consistent with that reported in literature. Of the 26 patients, 15 were culture positive for $E$. coli, and all but one patient was ciprofloxacin resistant. Of the $14 / 26$ patients who received extended ciprofloxacin for asymptomatic PSA elevation prior to biopsy, $12 / 14$ were resistant to ciprofloxacin. Interestingly, overall sepsis rate increased from $0.93 \%$ to $4.35 \%$ over 3 years. However, sepsis rate decreased after cessation of empiric extended treatment of asymptomatic elevated PSA, though the result was not statistically significant $(P=0.086)$.

Patel et al found that antibiotic use in the preceding 4 weeks of biopsy was independently associated with a four times greater risk of infection $(P=0.025)$. However, this was based on a relatively small sample size of four patients among the $16 / 300$ patients who developed infection and received antibiotics within the month of biopsy. Of the $10 / 16$ patients with culture positivity, all cases were due to fluoroquinoloneresistant E. coli. ${ }^{59}$

Risk of prior antibiotic use in post-prostate biopsy infection rate is relevant for a period of 6 months prior to biopsy. This was demonstrated in a recent prospective multicenter study of 2,718 patients, where the authors found that the OR of occurrence of post-prostate biopsy infection was 2.1 $(P=0.015) .{ }^{60}$ Overall, these studies highlight the importance of querying patient's recent antimicrobial usage and prompt the physician to tailor prophylactic regimen selection accordingly.

\section{Multidrug-resistant organisms}

\section{Fluoroquinolone-resistant organisms}

The prevalence of fluoroquinolone-resistant organisms in men undergoing PNB has varied between $12 \%$ and $25 \%$ in the literature. ${ }^{50-54,61}$ More current statistics on its prevalence in patients with infectious complications after biopsy are available from the Global Prevalence Study on Infections in Urology, which includes prospectively collected data from 702 men from 84 centers in Africa, Asia, Europe, and South America. The majority of patients (92.5\%) received fluoroquinolone prophylaxis prior to biopsy and 5.2\% developed symptomatic UTIs. Among the $37 \%$ of cases in which a causative bacteria was identified, $60 \%$ was attributed to fluoroquinolone resistance. ${ }^{4} \mathrm{~A}$ slightly higher rate of fluoroquinolone-resistant $E$. coli $(75 \%)$ was observed as a causative agent among patients presenting with urinary sepsis in a different single institution study. ${ }^{5}$

In a retrospective study of 1,273 patients, the overall incidence of post-prostate biopsy infections attributed to fluoroquinolone-resistant organisms was $1.2 \%(15 / 31$ cases). Furthermore, between 2004 and 2006, a statistically significant increase in both infectious complications and fluoroquinolone resistance was observed. ${ }^{7}$ Roberts et al found that an additional infection is incurred for every 14 men harboring fluoroquinolone-resistant organisms when compared to those without. $^{62}$

Several studies have showed benefit in the augmentation of standard prophylaxis with either amikacin or gentamicin where fluoroquinolone resistance is a concern. Infection rate decreased from $3.8 \%$ to $0.6 \%$ following augmentation with gentamicin, where only $9 \%$ resistance pattern was observed. ${ }^{55}$ When comparing to patients who received standard prophylaxis with either trimethoprim sulfamethoxazole 
or ciprofloxacin, this also saved US $\$ 15,700$ per 100 patients. Alternatively, amikacin-augmented fluoroquinolone prophylaxis significantly reduced septicemia rate, where fluoroquinolone-resistant $E$. coli was found to be responsible in $84.6 \%$ of cases; ${ }^{63}$ similar results were reported by Batura et al. ${ }^{64}$

\section{ESBL-producing organisms}

The ESBL-producing organisms constitute the other microbial class contributing to rising resistance against standard empiric prophylaxis in PNB. It appears that prevalence is increasing and attributing to increasing post-PNB infectious complications. A retrospective study performed in Sweden in 2000-2012 on 44,047 PNB cases showed an increase in positive blood cultures from $0.38 \%$ to $1.14 \%$ within 30 days of biopsy. Of the positive blood cultures, $8.3 \%$ were due to ESBL-producing enteric bacilli. The number of cases increased from two to 18 , before and after the year 2010 , respectively ${ }^{65}$ A prospective multicenter study conducted in Turkey showed that $19 \%$ of 400 men undergoing PNB carried ESBL-producing organisms. ${ }^{66}$ Presence was significantly associated with concurrent diabetes and previous fluoroquinolone usage $(P<0.05)$.

While carbapenems have been used as the mainstay of treatment for post-PNB infections secondary to ESBLproducing organisms, one study showed that in locations where resistance is high, augmentation with cefoxitin should be considered. ${ }^{67}$

\section{Recent travel}

A British study found that overseas travel was an independent risk factor for infectious complications (relative risk 2.7, $P=0.04) .{ }^{59}$ This is important historical information to solicit when initially counseling patients undergoing PNB. For example, Williamson et al performed a series analysis of 47 patients who developed $E$. coli bacteremia following prostate biopsy. Of these patients, $11 \%$ tested positive for ESBL variants. Among them, $4 / 5$ patients had traveled to India and Southeast Asia within the preceding month, areas where the CTX-M type of ESBLs is prevalent and confers resistance to both fluoroquinolones and aminoglycosides. ${ }^{68}$ Urologists may wish to tailor a patient's antimicrobial regimen according to these risk factors.

\section{Additional patient factors}

Studies have found associations between patient comorbidities and infection risk after PNB. Diabetes confers increased risk of febrile and infectious complications..$^{10,30,36,63,69}$ Practitioners may consider delaying prostate biopsy until glycemic control is adequate, although there have been no published studies on this measure. Hospitalization within the preceding month of biopsy is another known risk factor. ${ }^{36}$ Accordingly, a retrospective study by Kamdar et al showed that $75 \%$ of patients who developed post-PNB bacteremia were either health care employees or had a relative working in health care within their household. All of these patients carried fluoroquinolone-resistant E. coli. ${ }^{70}$ Influence of previous prostatitis history is debatable. ${ }^{10,60,63,69}$ Nonetheless, in patients with one or more of these risk factors, urologists may consider utilization of targeted prophylaxis or augmented antimicrobial therapy.

\section{Conclusion}

The risk of infectious complications has continued to rise likely owing to the increased incidence of drug-resistant organisms. Increasingly, however, studies have focused on different potential strategies to reduce the likelihood and severity of infections. Of particular benefit is that many of these approaches can be used in conjunction with one another as they focus on different elements of the biopsy process. As biopsy usage grows, prospective studies are certainly needed to better objectively evaluate the merits of different strategies.

\section{Disclosure}

The authors report no conflicts of interest in this work.

\section{References}

1. Society AC. Cancer Statistics. 2016. Available from: https://www. cancerstatisticscentercancerorg

2. Association AU. Early Detection of Prostate Cancer: American Urological Association AUA Guideline. May 2012:1-28.

3. FACS LGGM, MSCE EJHM, FACS EJTM. 109 - Prostate Biopsy: Techniques and Imaging. 11th ed. Elsevier Inc.; Philadelphia, PA, USA 2016: 2579-2592.e3.

4. Wagenlehner FME, van Oostrum E, Tenke P, et al. Infective complications after prostate biopsy: outcome of the Global Prevalence Study of Infections in Urology (GPIU) 2010 and 2011, a prospective multinational multicentre prostate biopsy study. Eur Urol. 2013;63(3):521-527.

5. Pinkhasov GI, Lin Y-K, Palmerola R, et al. Complications following prostate needle biopsy requiring hospital admission or emergency department visits - experience from 1000 consecutive cases. BJU Int. 2012;110(3):369-374.

6. Satyanarayana R, Parekh D. Prevention and treatment of biopsy-related complications. Curr Urol Rep. 2014;15(2):381-388.

7. Feliciano J, Teper E, Ferrandino M, et al. The incidence of fluoroquinolone resistant infections after prostate biopsy - are fluoroquinolones still effective prophylaxis? J Urol. 2008;179(3):952-955; discussion 955.

8. Carmignani L, Picozzi S, Spinelli M, et al. Bacterial sepsis following prostatic biopsy. Int Urol Nephrol. 2012;44(4):1055-1063.

9. Loeb S, Carter HB, Berndt SI, Ricker W, Schaeffer EM. Complications after prostate biopsy: data from SEER-Medicare. J Urol. 2011;186(5): 1830-1834.

10. Loeb S, van den Heuvel S, Zhu X, Bangma CH, Schröder FH, Roobol MJ. Infectious complications and hospital admissions after prostate biopsy in a European randomized trial. Eur Urol. 2012;61(6):1110-1114. 
11. Nam RK, Saskin R, Lee Y, et al. Increasing hospital admission rates for urological complications after transrectal ultrasound guided prostate biopsy. J Urol. 2010;183(3):963-969.

12. American Urological Association. AUA/optimal techniques of prostate biopsy and specimen handling. AUA Guideline. March 2013:1-29.

13. Isbarn H, Briganti A, De Visschere PJ, et al. Systematic ultrasoundguided saturation and template biopsy of the prostate: indications and advantages of extended sampling. Arch Esp Urol. 2015;68(3):296-306.

14. Ploussard G, Nicolaiew N, Marchand C, et al. Risk of repeat biopsy and prostate cancer detection after an initial extended negative biopsy: longitudinal follow-up from a prospective trial. BJU Int. 2013;111(6):988-996.

15. Pepe P, Dibenedetto G, Gulletta M, et al. Prostate cancer detection after one or more negative extended needle biopsy: results of a multicenter case-findings protocol. Arch Ital Urol Androl. 2010;82(2):95-99.

15. Berger AP, Gozzi C, Steiner H, et al. Complication Rate of transrectal ultrasound guided prostate biopsy: a comparison among 3 protocols with 6, 10 and 15 cores. J Urol. 2004;171(4):1478-1481.

16. Jeon SS, Woo S-H, Hyun J-H, Choi HY, Chai SE. Bisacodyl rectal preparation can decrease infectious complications of transrectal ultrasound-guided prostate biopsy. Urology. 2003;62(3):461-466.

17. Pepe $\mathrm{P}$, Aragona F. Morbidity after transperineal prostate biopsy in 3000 patients undergoing 12 vs 18 vs more than 24 needle cores. Urology. 2013;81(6):1142-1146.

18. Loeb S, Carter HB, Berndt SI, Ricker W, Schaeffer EM. Is repeat prostate biopsy associated with a greater risk of hospitalization? Data from SEER-Medicare. J Urol. 2013;189(3):867-870.

20. Lindert KA, Kabalin JN, Terris MK. Bacteremia and bacteriuria after transrectal ultrasound guided prostate biopsy. J Urol. 2000;164(1):76-80.

21. Grummet JP, Weerakoon M, Huang S, et al. Sepsis and "superbugs": should we favour the transperineal over the transrectal approach for prostate biopsy? BJU Int. 2014;114(3):384-388.

22. Cerruto MA, Vianello F, D'Elia C, Artibani W, Novella G. Transrectal versus transperineal 14-core prostate biopsy in detection of prostate cancer: a comparative evaluation at the same institution. Arch Ital Urol Androl. 2014;86(4):284-287.

23. Bjurlin MA, Wysock JS, Taneja SS. Optimization of prostate biopsy. Urol Clin N Am. 2014;41(2):299-313.

24. Tuncel A, Aslan Y, Sezgin T, Aydin O, Tekdogan U, Atan A. Does disposable needle guide minimize infectious complications after transrectal prostate needle biopsy? Urology. 2008;71(6):1024-1027; discussion 1027-1028

25. Gurbuz C, Canat L, Atis G, Caskurlu T. Reducing infectious complications after transrectal prostate needle biopsy using a disposable needle guide: is it possible? Int Braz J Urol. 2011;37(1):79-84; discussion 85-86.

26. Issa MM, Al-Qassab UA, Hall J, Ritenour CWM, Petros JA, Sullivan JW. Formalin disinfection of biopsy needle minimizes the risk of sepsis following prostate biopsy. J Urol. 2013;190(5):1769-1775.

27. Koc G, Un S, Filiz DN, Akbay K, Yilmaz Y. Does washing the biopsy needle with povidone-iodine have an effect on infection rates after transrectal prostate needle biopsy? Urol Int. 2010;85(2):147-151.

28. Lorber G, Duvdevani M, Friedman M, et al. Sustained-release antibacterial varnish-coated biopsy needle for reduction of infection rates following prostate biopsy-in vitro model. J Endourol. 2013;27(3): 277-283.

29. Shandera KC, Thibault GP, Deshon GE. Variability in patient preparation for prostate biopsy among American urologists. Urology. 1998;53(4):644-646.

30. Carey JM, Korman HJ. Transrectal ultrasound guided biopsy of the prostate. Do enemas decrease clinically significant complications? $J$ Urol. 2001;166(1):82-85.

31. Vallancien G, Prapotnich D, Veillon B, Brisset JM, Andre-Bougaran J. Systematic prostatic biopsies in 100 men with no suspicion of cancer on digital rectal examination. J Urol. 1991;146(5):1308-1312.

32. Ruddick F, Sanders P, Bicknell SG, Crofts P. Sepsis rates after ultrasound-guided prostate biopsy using a bowel preparation protocol in a community hospital. J Ultrasound Med. 2011;30(2):213-216.
33. Abughosh Z, Margolick J, Goldenberg SL, et al. A prospective randomized trial of povidone-iodine prophylactic cleansing of the rectum before transrectal ultrasound guided prostate biopsy. J Urol. 2013;189(4):1326-1331.

34. Kanjanawongdeengam P, Viseshsindh W, Santanirand P, Prathombutr P, Nilkulwattana $\mathrm{S}$. Reduction in bacteremia rates after rectum sterilization before transrectal, ultrasound-guided prostate biopsy: a randomized controlled trial. J Med Assoc Thai. 2009;92(12):1621-1626.

35. Gyorfi JR, Otteni C, Brown K, et al. Peri-procedural povidone-iodine rectal preparation reduces microorganism counts and infectious complications following ultrasound-guided needle biopsy of the prostate. World J Urol. 2014;32(4):905-909.

36. Carignan A, Roussy J-F, Lapointe V, Valiquette L, Sabbagh R, Pépin J. Increasing risk of infectious complications after transrectal ultrasoundguided prostate biopsies: time to reassess antimicrobial prophylaxis? Eur Urol. 2012;62(3):453-459.

37. Burden HP, Ranasinghe W, Persad R. Antibiotics for transrectal ultrasonography-guided prostate biopsy: are we practising evidence-based medicine? BJU Int. 2008;101(10):1202-1204.

38. Aron M, Rajeev TP, Gupta NP. Antibiotic prophylaxis for transrectal needle biopsy of the prostate: a randomized controlled study. BJU Int. 2000;85(6):682-685.

39. Kapoor DA, Klimberg IW, Malek GH, et al. Single-dose oral ciprofloxacin versus placebo for prophylaxis during transrectal prostate biopsy. Urology. 1998;52(4):552-558.

40. Crawford ED, Haynes AL, Story MW, Borden TA. Prevention of urinary tract infection and sepsis following transrectal prostatic biopsy. J Urol. 1982;127(3):449-451

41. Brown RW, Warner JJ, Turner BI, Harris LF, Alfort RH. Bacteremia and bacteriuria after transrectal prostatic biopsy. Urology. 1981;18(2): $145-148$.

42. Naber KG, Sorgel F. Antibiotic therapy-rationale and evidence for optimal drug concentrations in prostatic and seminal fluid and in prostatic tissue. Andrologia. 2003;35(5):331-335.

43. Wolf JS, Bennett CJ, Dmochowski RR, Hollenbeck BK, Schaeffer AJ. Urologic surgery antimicrobial prophylaxis. American Urological Association Best Practice Policy Statement. 2014:1-48.

44. Zani EL, Clark OAC, Rodrigues Netto N. Antibiotic prophylaxis for transrectal prostate biopsy. Cochrane Database Syst Rev. 2011;(5):CD006576.

45. Mrkobrada M, Ying I, Mokrycke S, et al. CUA guidelines on antibiotic prophylaxis for urologic procedures. CUAJ. 2015;9(1-2):1-10.

46. EAU Guidelines on Prostate Cancer. March 2015:1-137.

47. European Association of Urology Guidelines. March 2016:1-1159.

48. Shigemura K, Tanaka K, Yasuda M, et al. Efficacy of 1-day prophylaxis medication with fluoroquinolone for prostate biopsy. World J Urol. 2005;23(5):356-360

49. Sabbagh R, McCormack M, Péloquin F, et al. A prospective randomized trial of 1-day versus 3-day antibiotic prophylaxis for transrectal ultrasound guided prostate biopsy. Can J Urol. 2004;11(2):2216-2219.

50. Duplessis CA, Bavaro M, Simons MP, et al. Rectal cultures before transrectal ultrasound-guided prostate biopsy reduce post-prostatic biopsy infection rates. Urology. 2012;79(3):556-561.

51. Taylor AK, Zembower TR, Nadler RB, et al. Targeted antimicrobial prophylaxis using rectal swab cultures in men undergoing transrectal ultrasound guided prostate biopsy is associated with reduced incidence of postoperative infectious complications and cost of care. J Urol. 2012;187(4):1275-1279.

52. Dai J, Leone A, Mermel L, et al. Ambulatory, office-based, and geriatric urology rectal swab culture-directed antimicrobial prophylaxis for prostate biopsy and risk of postprocedure infection: a cohort study. Urology. 2015;85(1):8-14.

53. Summers SJ, Patel DP, Hamilton BD, et al. An antimicrobial prophylaxis protocol using rectal swab cultures for transrectal prostate biopsy. World J Urol. 2015;33(12):2001-2007.

54. Liss MA, Kim W, Moskowitz D, Szabo RJ. Comparative effectiveness of targeted vs empirical antibiotic prophylaxis to prevent sepsis from transrectal prostate biopsy: a retrospective analysis. J Urol. 2015;194(2):397-402. 
55. Adibi M, Pearle MS, Lotan Y. Cost-effectiveness of standard vs intensive antibiotic regimens for transrectal ultrasonography (TRUS)-guided prostate biopsy prophylaxis. BJU Int. 2012;110(2 Pt 2):E86-E91.

56. Li C-K, Tong BCY, You JHS. Cost-effectiveness of culture-guided antimicrobial prophylaxis for the prevention of infections after prostate biopsy. Int J Infect Dis. 2016;43:7-12.

57. Akduman B, Akduman D, Tokgöz H, et al. Long-term fluoroquinolone use before the prostate biopsy may increase the risk of sepsis caused by resistant microorganisms. Urology. 2011;78(2):250-255.

58. Lodeta B, Trkulja V. Septic complications and hospital admissions after transrectal ultrasound-guided prostate biopsy: incidence rates and outcomes in 913 consecutive biopsies. Int Urol Nephrol. 2014;46(12): 2335-2336.

59. Patel U, Dasgupta P, Amoroso P, Challacombe B, Pilcher J, Kirby R. Infection after transrectal ultrasonography-guided prostate biopsy: increased relative risks after recent international travel or antibiotic use. BJU Int. 2012;109(12):1781-1786.

60. Bruyère F, Malavaud S, Bertrand P, et al. Prosbiotate: a multicenter, prospective analysis of infectious complications after prostate biopsy. JUrol. 2015;193(1):145-150.

61. Cohen JE, Landis P, Trock BJ, et al. Fluoroquinolone resistance in the rectal carriage of men in an active surveillance cohort: longitudinal analysis. J Urol. 2015;193(2):552-556.

62. Roberts MJ, Williamson DA, Hadway P, Doi SAR, Gardiner RA, Paterson DL. Baseline prevalence of antimicrobial resistance and subsequent infection following prostate biopsy using empirical or altered prophylaxis: a bias-adjusted meta-analysis. Int JAntimicrob Agents. 2014;43(4):301-309.
63. Kehinde EO, Al-Maghrebi M, Sheikh M, Anim JT. Combined ciprofloxacin and amikacin prophylaxis in the prevention of septicemia after transrectal ultrasound guided biopsy of the prostate. J Urol. 2013;189(3):911-915.

64. Batura D, Rao GG, Bo Nielsen P, Charlett A. Adding amikacin to fluoroquinolone-based antimicrobial prophylaxis reduces prostate biopsy infection rates. BJU Int. 2011;107(5):760-764.

65. Aly M, Dyrdak R, Nordström T, et al. Rapid increase in multidrugresistant enteric bacilli blood stream infection after prostate biopsy - a 10-year population-based cohort study. Prostate. 2015;75(9):947-956.

66. Tukenmez Tigen E, Tandogdu Z, Ergonul O, et al. Outcomes of fecal carriage of extended-spectrum $\beta$-lactamase after transrectal ultrasoundguided biopsy of the prostate. Urology. 2014;84(5):1008-1015.

67. Horcajada JP, Busto M, Grau S, et al. High prevalence of extendedspectrum beta-lactamase-producing enterobacteriaceae in bacteremia after transrectal ultrasound-guided prostate biopsy: a need for changing preventive protocol. Urology. 2009;74(6):1195-1199.

68. Williamson DA, Masters J, Freeman J, Roberts S. Travel-associated extended-spectrum $\beta$-lactamase-producing Escherichia coli bloodstream infection following transrectal ultrasound-guided prostate biopsy. $B J U$ Int. 2012;109(7):E21-E22.

69. Schaeffer AJ, Montorsi F, Scattoni V, et al. Comparison of a 3-day with a 1-day regimen of an extended-release formulation of ciprofloxacin as antimicrobial prophylaxis for patients undergoing transrectal needle biopsy of the prostate. BJU Int. 2007;100(1):51-57.

70. Kamdar C, Mooppan UMM, Gulmi FA, Kim H. Multi-drug-resistant bacteremia after transrectal ultrasound guided prostate biopsies in hospital employees and their relatives. Urology. 2008;72(1):34-36.
Infection and Drug Resistance

\section{Publish your work in this journal}

Infection and Drug Resistance is an international, peer-reviewed openaccess journal that focuses on the optimal treatment of infection (bacterial, fungal and viral) and the development and institution of preventive strategies to minimize the development and spread of resistance. The journal is specifically concerned with the epidemiology of antibiotic

\section{Dovepress}

resistance and the mechanisms of resistance development and diffusion in both hospitals and the community. The manuscript management system is completely online and includes a very quick and fair peerreview system, which is all easy to use. Visit http://www.dovepress.com/ testimonials.php to read real quotes from published authors. 